\title{
Correction to: FabG: from a core to circumstantial catalyst
}

\author{
Anirudh P. Shanbhag
}

Published online: 17 May 2019

(C) Springer Nature B.V. 2019

\section{Correction to: Biotechnol Lett}

https://doi.org/10.1007/s10529-019-02678-2

In the original publication of the article, under section Polyketide synthesis: a pathway similar to fatty acid synthesis, the sentences "Phylogeny of KS domains and proteins of FAS and PKS, inferred by Bayesian estimation. Numbers above branches indicate posterior clade probability values." and "Branch length indicates number of inferred amino acid changes per position." in the first paragraph were included inadvertently.

The corrected paragraph is given below.

Polyketide synthesis is a natural process present in a few bacteria and most of the fungi for producing toxins and antibiotics. In an industrial perspective it is used for the production of antibiotic, antitumor, antifungal, and immunosuppressive compounds. This is done with the help of polyketide synthase (PKS) enzyme complex. It has evolved in both bacteria and fungi for synthesizing various secondary metabolites. There is an implication of the structural and functional similarities between PKS and FAS in both prokaryotes and eukaryotes. Phylogenetic studies show that the FAS II and PKSs II systems are related, and have existed through horizontal gene transfer. There are three types of polyketide synthase (PKS) complex like the FAS. Type I PKS is a single modular protein similar to FASI whereas the PKS II is a set of enzymes which perform carbon chain elongation like FAS II. However, the third type of PKS doesn't have ACP binding domains.

The original article can be found online at https://doi.org/10.1007/s10529-019-02678-2.

A. P. Shanbhag

Department of Biophysics, Molecular Biology and Bioinformatics, University of Calcutta, Kolkata 700009, India

A. P. Shanbhag ( $\square)$

Bugworks Research India Pvt. Ltd, C-CAMP, NCBS

Campus, UAS-GKVK, Bellary Road, Bangalore 560065,

India

e-mail: anirudh@bugworksresearch.com 